\title{
Hyperammonemia as a Marker of Subclinical Seizures After Traumatic Cardiac Arrest
}

\author{
Patricia Aswani, ${ }^{1}$ and Michael Kalina ${ }^{2, *}$ \\ ${ }^{1}$ Department of Research, Staten Island University Hospital, New York, United States \\ ${ }^{2}$ Department of Surgery, Staten Island University Hospital, New York, United States \\ "Corresponding author: Michael Kalina, Department of Surgery, Staten Island University Hospital, Director of Emergency Surgery, 256 Mason Avenue, Suite C Staten Island, \\ New York, United States. Tel: +718-2256398, Fax:+718-2261247, E-mail: mkalina@nshs.edu
}

Received 2015 September 23; Revised 2015 December 07; Accepted 2015 December 11.

\begin{abstract}
This report details the presence of hyperammonemia in a patient who sustained cardiac arrest after a traumatic amputation. Serum ammonia levels may rise due to numerous etiologies; however, few reports detail its usefulness in diagnosing subclinical seizures. In this case, we successfully utilized persistently elevated serum ammonia levels as a marker of subclinical seizures in a patient who sustained traumatic cardiac arrest.
\end{abstract}

Keywords: Hyperammonemia, Seizure, Traumatic Cardiac Arrest

\section{Introduction}

This report details the presence of hyperammonemia in a patient who sustained cardiac arrest after a traumatic amputation. Serum ammonia levels may rise due to numerous etiologies; however, few reports detail its usefulness in diagnosing subclinical seizures.

\section{Case Presentation}

A 51-year- old male presented to the emergency department (ED) in hemorrhagic shock from a traumatic leg amputation after he was struck by a car. The patient had a glasgow coma score (GCS) of 3 and sustained a cardiac arrest. In the ED, the hemorrhage was controlled, blood products were administered, and the patient had a rapid return of spontaneous circulation. The patient was taken to the operating room for definitive hemorrhage control and completion amputation. Head computerized tomography (CT) scan revealed cerebral edema. Keppra (Levetiracetam) $500 \mathrm{mg}$ was given twice daily for seizure prophylaxis, mannitol 12.5 grams was given every eight hours for cerebral edema and an intracranial pressure (ICP) monitor was placed. Initial labs included $\mathrm{pH}$ 6.8, lactate $>15$ mmol/L, creatinine kinase (CK) 1267 IU/L, aspartate aminotransferase (AST) $354 \mathrm{IU} / \mathrm{L}$, alanine aminotransferase (ALT) $391 \mathrm{IU} / \mathrm{L}$, and total bilirubin (TB) $0.6 \mathrm{mg} / \mathrm{dL}$. Day 2, repeat labs revealed $\mathrm{pH} 7.44$ and lactate $1.6 \mathrm{mmol} / \mathrm{L}$. CK levels rose to $1761 \mathrm{IU} / \mathrm{L}$ but trended down to $887 \mathrm{IU} / \mathrm{L}$ by day 4 . Hepatic transaminases peaked AST 7163 IU/L and ALT 5541 IU/L day 2, normalizing by day 7 (Figure 1). Repeat neurologic assessments revealed GCS of 3 with a cough, gag reflex, and spontaneous respirations on the mechanical ventilator. Repeat head CT scan revealed cerebral edema with evidence of anoxic encephalopathy. An electroencephalogram (EEG) revealed frequent diffusely expressed generalized spikes representative of seizure activity, despite no generalized seizures. Serum ammonia level was elevated, $37 \mu \mathrm{mol} / \mathrm{L}$. Keppra dosing was increased to $1000 \mathrm{mg}$ given twice daily and the subclinical seizure activity on EEG resolved. Days 4 - 8, the ICP monitor was removed and there was no change in neurologic status. Despite lactulose administration, the serum ammonia level remained elevated. Therefore, a repeat EEG was performed, as seizures may be a source of the hyperammonemia. This EEG revealed an increased frequency of spikes, creating a pattern suggestive of seizures. Keppra dosing was increased to $1500 \mathrm{mg}$ given twice daily. Dilantin (Phenytoin) 100 mg was given every 8 hours in addition to Keppra, with resolution of the subclinical seizure activity on EEG. Day 10, the patient underwent tracheostomy and percutaneous endoscopic gastrostomy tube placement. Day 18, the patient transferred to the surgical ward and was discharged to traumatic brain injury rehabilitation day 32. Discharge GCS was 5, repeat EEG's revealed no seizure activity, and the serum ammonia level normalized. 


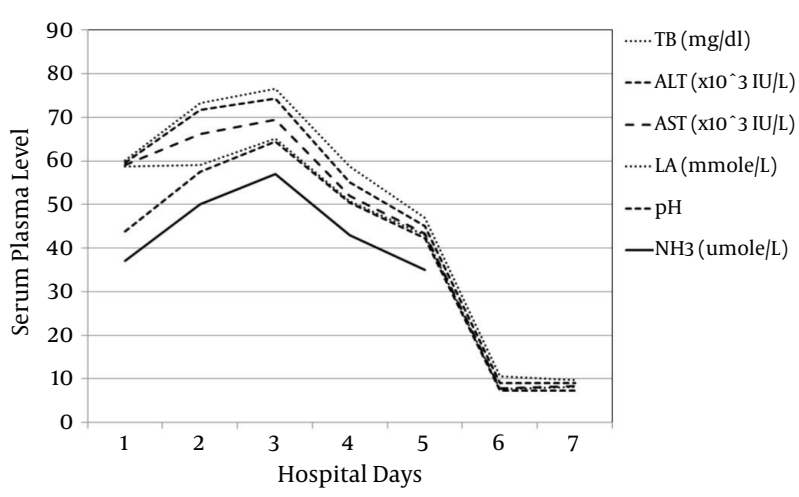

Figure 1. Laboratory Data

\section{Discussion}

Ammonia is toxic to the central nervous system and can lead to personality changes, loss of consciousness, neuromuscular dysfunction, cerebral edema, diminished cognition, coma, and death. Most commonly, hyperammonemia is derived from liver cirrhosis, a dysfunctional liver unable to metabolize ammonia leading to its accumulation. If the liver functions normally, then ammonia should be cleared. Therefore, in the non-cirrhotic patient, hyperammonemia may derive from medication toxicities, urea cycle disorders, infection due to urease-producing bacteria, errors in metabolism, hyperalimentation, trauma, acidosis, increased muscle activity, and renal tubular acidosis (1). Nakamura et al. found a strong correlation between ammonia elevation and acidosis in idiopathic epileptic patients. They concluded that the mechanism for hyperammonemia after seizure was attributed to ammonia produced from acidosis and from muscle contraction. Acidosis can inhibit the glycolysis pathway in red blood cells, thereby leading to an accumulation of ammonia in the blood (2). Barta and Babusikova suggested that ammonia in cardiac arrest patients was produced mainly in red blood cells due to acidosis and hypoxia (3). Lin et al. investigated the association between elevated blood ammonia and partial pressure of ammonia seen in out of hospital cardiac arrest patients and their return to spontaneous circulation (ROSC). They found that blood ammonia levels greater than $84 \mathrm{umol} / \mathrm{L}$ were most predictive of patients who would did not have ROSC (4). Hyperammonemia from seizures can derive from excessively contracting muscles. Generalized tonic-clonic seizures are the only seizure type reported associated with excessive production of ammonia. The contractions from a generalized seizure lead to muscle protein breakdown and the accumulation of ammonia in the blood. This rapid accumulation of ammo- nia overwhelms the liver's ability to metabolize it. However, once the seizures cease, a healthy liver should rapidly lower ammonia levels. This mechanism is emphasized by the excess of serum ammonia found in non-cirrhotic patients after strenuous exercise (5). Hung and colleagues proposed that transient hyperammonemia is more of a consequence rather than a primary cause of seizures. They suggested that if hyperammonemia was the primary cause of seizures, ammonia levels should remain elevated until therapy is initiated (6). This report details persistently elevated serum ammonia levels in a non-cirrhotic trauma patient who had resolution of numerous potential etiologies for the hyperammonemia including cardiac arrest, severe metabolic acidosis, ischemic hepatitis, and rhabdomyolysis. In this case, the etiology for hyperammonemia was persistent subclinical seizures. With aggressive use of seizure medications, the seizures were controlled at which time the serum ammonia levels normalized.

The etiology of hyperammonemia can be multifactorial in the non-cirrhotic patient. This report is the first to detail the use of hyperammonemia as a marker of subclinical seizures in a trauma patient post cardiac arrest.

\section{Footnote}

Authors' Contribution: Patricia Aswani, data acquisition, manuscript production; Michael Kalina, data acquisition, manuscript production and revision.

\section{References}

1. LaBuzetta JN, Yao JZ, Bourque DL, Zivin J. Adult nonhepatic hyperammonemia: a case report and differential diagnosis. Am J Med. 2010;123(10):885-91. doi:10.1016/j.amjmed.2010.02.029. [PubMed: 20920686].

2. Nakamura K, Yamane K, Shinohara K, Doi K, Inokuchi R, Hiruma T, et al. Hyperammonemia in idiopathic epileptic seizure. Am J Emerg Med. 2013;31(10):1486-9. doi: 10.1016/j.ajem.2013.08.003. [PubMed: 24007697].

3. Barta E, Babusikova F. The concentration of ammonia in blood and plasma stored for transfusion. Resuscitation. 1982;10(2):135-9. [PubMed: 6294769].

4. Lin $\mathrm{CH}$, Chi CH, Wu SY, Hsu HC, Chang YH, Huang YY, et al. Prognostic values of blood ammonia and partial pressure of ammonia on hospital arrival in out-of-hospital cardiac arrests. Am JEmerg Med. 2013;31(1):8-15. doi: 10.1016/j.ajem.2012.04.037. [PubMed: 22795429].

5. Liu KT, Yang SC, Yeh IJ, Lin TJ, Lee CW. Transient hyperammonemia associated with postictal state in generalized convulsion. Kaohsiung J Med Sci. 2011;27(10):453-6. doi: 10.1016/j.kjms.2011.06.005. [PubMed: 21943818].

6. Hung TY, Chen CC, Wang TL, Su CF, Wang RF. Transient hyperammonemia in seizures: a prospective study. Epilepsia. 2011;52(11):2043-9. doi: 10.1111/j.1528-1167.2011.03279.x. [PubMed: 21972984]. 